\title{
Single-Session Lung Radiosurgery Using Robotic Image-Guided Real-Time Respiratory Tumor Tracking
}

\author{
Alexander Muacevic ${ }^{1}$, Markus Kufeld ${ }^{2}$, B. Wowra ${ }^{3}$, H. Winter ${ }^{4}$, H. T. Hofmann ${ }^{5}$ \\ 1. European CyberKnife Center Munich 2. Charité Cyberknife Center Berlin 3. European Cyberknife \\ Center Munich-Grosshadern 4. Department of Thoracic Surgery, University of Munich Hospital 5. \\ Institute of Clinical Radiology, University of Munich Hospital
}

$\square$ Corresponding author: Alexander Muacevic, alexander.muacevic@cureus.com Disclosures can be found in Additional Information at the end of the article

\section{Abstract}

Objective: To describe the clinical feasibility for single session lung radiosurgery using a realtime robotic system with respiratory tracking.

Methods: We treated 62 patients with 75 lung tumors with robotic radiosurgery (CyberKnife ${ }^{\circledR}$, Accuray). Twenty-two patients had primary tumors and 40 had metastatic tumors. Forty-two patients underwent computed tomography-guided percutaneous placement of one fiducial directly into the tumor. Ten patients were treated using spinal tracking, and in another ten patients fiducial free lung tumor tracking (Xsight lung, Accuray Inc.) was performed. All patients were treated with single session radiosurgery to a dose of $22-24 \mathrm{~Gy}$. The median tumor volume was 15 cc $(1.5-64 \mathrm{cc})$. The last 23 patients were planned with a new Monte Carlo treatment planning algorithm. Follow-up CT scanning was performed every two months.

Results: All patients could be treated with the automated robotic technique. The 6-, 12-, 24 actuarial local tumor control rate was $98 \%, 88 \%$ and $88 \%$, respectively. The median follow up time was 10 months (range: 0.4 - 30 months). Six patients (10\%) required a chest tube insertion after fiducial implantation because of pneumothorax. Four patients experienced nausea after treatment. No other short-term adverse reactions were found. Fifteen patients showed Grade 1 and one patient Grade 2 signs of pneumonitis. No Grade 3 or grade 4 toxicity was detected.

Conclusions: Single-session radiosurgery for lung tumor tracking using the described technology is a stable, safe, and feasible concept for respiratory tracking of tumors during robotic lung radiosurgery in selected patients. The single fraction approach is convenient for the patient and does not interfere with concomitant systemic treatments. Longer follow-up is needed for definitive clinical results.

Published 12/09/2009

(๑) Copyright 2009

Muacevic et al. This is an open access article distributed under the terms of the Creative Commons Attribution License CC-BY 3.0., which permits unrestricted use, distribution, and reproduction in any medium, provided the original author and source are credited.
Categories: Radiation Oncology

Keywords: cyberknife, lung cancer, radiosurgery, robotics, image guided robotics, lung radiosurgery

\section{Introduction}

The preferred treatment for patients with non-small cell lung cancer (NSCLC) or single lung metastases from other cancers remains surgery. However, comorbidities, low performance status, poor lung function, or previous surgery often prohibit additional surgical approaches, so alternative treatment options have to be selected. In most of these inoperable patients, 
conventional radiation therapy, which can result in local control rates of $40-70 \%$, or palliative chemotherapy is chosen [1-4]. Conventional, conformal radiation therapy in the lung is made technically difficult by the substantial and variable target motion in this organ. Often, the total dose needed to destroy the cancerous tissue cannot be fully applied while protecting the surrounding healthy tissue. This problem has been addressed in a number of ways, including immobilizing the patient using body frames and abdominal compression devices [5-6]. Recently, gating techniques have been developed that allow the treatment beam to be turned on when breathing brings the tumor to within a predefined range and turned off when it leaves this target range. Although each of these techniques has permitted substantial dose escalation, they also require considerable set-up margins to assure that the tumor receives sufficient radiation. A consequence is that a significant amount of healthy tissue is irradiated.

Accordingly, most published series on stereotactic lung radiotherapy report hypofractionated treatment regimes [5-7].

We believe that the aim of lung radiosurgery should be the same as the aim of brain radiosurgery, that is, to maximize radiation to the tumor and minimize radiation exposure to surrounding normal tissue and adjacent critical structures. The newly developed respiratory tracking system that is a feature of the CyberKnife ${ }^{\circledR}$ System (Synchrony ${ }^{\circledR}$ Respiratory Tracking, Accuray Inc., Sunnyvale, CA) should allow achievement of this goal. The integrated imaging system coupled to the robotically-controlled linear accelerator is designed to precisely and dynamically track moving targets, such as pulmonary tumors. It is the purpose of the current paper to describe the clinical application using a stringent and minimally invasive setup for selected patients with NSCLC or pulmonary metastases.

\section{Materials And Methods}

\section{Radiosurgery system}

The CyberKnife robotic radiosurgery system consists of a 6-MV compact linear accelerator (LINAC) mounted on a computer-controlled six-axis robotic manipulator. Integral to the system are orthogonally positioned $x$-ray cameras which acquire images during treatment. The images are processed automatically to identify radiographic features, and are registered to the treatment planning study to measure the position of the treatment site in real time. The system adapts to changes in patient position during treatment by acquiring targeting images repeatedly and adjusting the direction of the treatment beam accordingly. The treatment beam can be directed from hundreds of angles anterior and lateral to the patient.

\section{Respiratory motion compensation}

In order to compensate for tumor motion in the lung, it is necessary to know the internal position of the target throughout treatment. At any given time, the stereo X-ray system can acquire images to establish the tumor position. Conventionally, small gold fiducials implanted in the proximity of the target are identified in both images and used to compute the 3D target position. While this provides accurate information on the tumor location at the instant the Xrays are taken, this is not sufficient to obtain real-time motion information. In the present system, the position of the chest is measured continuously by an external camera array that tracks the position of infrared markers on the patient's chest, and the location of the tumor is detected periodically in the x-ray images. A series of internal and external position measurements taken at the same time is used to establish a correlation model. Throughout treatment, occasional measurements of both positions are used to check and update the model.

The correlation model allows the internal tumor position to be estimated based on the external marker position. Because the frequency of the external camera system is approximately 30 Hertz, this estimation can occur almost continuously while keeping the diagnostic X-ray dose 
low. Nevertheless, data processing and robot motion cause a time lag $\Delta \mathrm{t}$. While this lag is small, it can be handled based on the cyclic nature of respiratory motion. At each time step $t_{i}$, the position of the tumor at time $t_{i}+\Delta t$ is computed. The robot is then controlled, such that the beam will always point to the tumor [8-10].

Fiducial-free tracking was done using the Xsight Lung Tracking (XLT, Accuray, Inc.) software which uses the actual image intensity of the lung tumor for targeting the beam. and therefore, eliminates the need for fiducial implantation. XLT uses advanced image processing and image registration techniques to automatically lock onto the tumor directly, tracking it throughout the procedure. The XLT System is able to track most peripherally located lung tumors that are at least $15 \mathrm{~mm}$ in diameter as it relies on the difference of the tissue density between the tumor and the surrounding lung tissue.

\section{Patients}

Sixty-two patients were treated for 77 lung tumors with radiosurgery (Table 1). There were 35 men and 27 women. The age range was 22 to 87 years (mean age: 63 years). Twentytwo patients had primary lung tumors and 40 had metastatic tumors. One patient underwent radiosurgery for four lesions and eleven for two lesions. All others were treated for a single tumor. We only selected patients with a maximal tumor diameter of $3.5 \mathrm{~cm}$. Nine patients (14\%) underwent prior conventional radiation therapy for the targeted lesion. Twenty-three patients received chemotherapy before or during SRS treatment and 17 patients underwent surgery before SRS. Tumor volume ranged from 1.5 to $64 \mathrm{cc}$ (median: $15 \mathrm{cc}$ ).

\begin{tabular}{|c|c|c|c|c|c|c|c|c|}
\hline Pat. Nr. & No. treat. & Sex & Age (y) & Primary & Volume & Dose & Isodose & 10 Gy \\
\hline 1 & & $f$ & 57 & BC & 12,4 & 24 & 70 & 26.96 \\
\hline 2 & & $f$ & 71 & CR & 64,2 & 22 & 75 & 250.2 \\
\hline 3 & & $\mathrm{~m}$ & 71 & CR & 22,7 & 24 & 75 & 92.9 \\
\hline \multirow[t]{2}{*}{4} & 2 & $\mathrm{~m}$ & 53 & Pharynx & 21,7 & 24 & 80 & 340.6 \\
\hline & & $\mathrm{m}$ & 53 & Pharynx & 29,9 & 24 & 80 & 234.3 \\
\hline 5 & & $\mathrm{~m}$ & 65 & $B C$ & 4,1 & 24 & 75 & 52.4 \\
\hline 6 & & $f$ & 62 & BC & 17,2 & 24 & 70 & 132.99 \\
\hline 7 & & $f$ & 77 & Other & 23,5 & 24 & 70 & 138.46 \\
\hline 8 & & $\mathrm{~m}$ & 68 & BC & 11,3 & 24 & 70 & 49.44 \\
\hline 9 & & $\mathrm{~m}$ & 59 & BC & 26,7 & 24 & 75 & 145.2 \\
\hline 10 & & $f$ & 86 & UGT & 39,3 & 24 & 70 & 213.2 \\
\hline 11 & & $\mathrm{~m}$ & 54 & $B C$ & 8,1 & 24 & 70 & 56.11 \\
\hline 12 & & $\mathrm{~m}$ & 44 & Melanoma & 56,6 & 24 & 70 & 223.83 \\
\hline 13 & & $\mathrm{~m}$ & 66 & $B C$ & 4,8 & 24 & 75 & 31.54 \\
\hline 14 & & $f$ & 59 & $B C$ & 2,3 & 24 & 75 & 14.54 \\
\hline
\end{tabular}




\section{Cureus}

\begin{tabular}{|c|c|c|c|c|c|c|c|c|}
\hline 15 & & $\mathrm{~m}$ & 22 & UGT & 1,5 & 24 & 75 & 9.89 \\
\hline 16 & & m & 73 & BC & 5,4 & 24 & 75 & 37.15 \\
\hline 17 & 2 & $\mathrm{~m}$ & 53 & BC & 23,8 & 24 & 75 & 229.83 \\
\hline & & $\mathrm{m}$ & 53 & BC & 20,8 & 24 & 75 & 229.83 \\
\hline 18 & & $\mathrm{~m}$ & 63 & CR & 9,7 & 24 & 75 & 49.09 \\
\hline 19 & & $f$ & 71 & BC & 13,1 & 24 & 75 & 68.84 \\
\hline 20 & & $\mathrm{~m}$ & 78 & BC & 5,7 & 24 & 75 & 38.47 \\
\hline 21 & & $\mathrm{~m}$ & 78 & BC & 47,1 & 24 & 75 & $161.8 \mathrm{~s}$ \\
\hline 22 & & $f$ & 66 & UGT & 23,14 & 24 & 65 & 74.1 \\
\hline 23 & & $f$ & 66 & BC & 14,6 & 24 & 75 & 81.06 \\
\hline 24 & & $\mathrm{~m}$ & 63 & BC & 30,2 & 24 & 75 & 150.4 \\
\hline 25 & & $f$ & 59 & BC & 8,4 & 24 & 75 & 39.06 \\
\hline 26 & & $f$ & 79 & Pharynx & 15,2 & 24 & 75 & 78.03 \\
\hline 27 & & m & 57 & CR & 11,3 & 24 & 70 & 75 \\
\hline 28 & & $f$ & 77 & BC & 49,2 & 24 & 75 & 225.2 \\
\hline 29 & & $\mathrm{~m}$ & 68 & CR & 20,8 & 24 & 60 & 145.5 \\
\hline 30 & 2 & $\mathrm{~m}$ & 59 & GIT & 15,2 & 24 & 65 & 76.2 \\
\hline & & $\mathrm{m}$ & 59 & GIT & 11,2 & 24 & 65 & 101.5 \\
\hline 31 & 2 & $\mathrm{~m}$ & 77 & CR & 19,8 & 24 & 75 & 160 \\
\hline & & m & 77 & CR & 4,3 & 24 & 75 & 160 \\
\hline 32 & & $f$ & 21 & Sarkoma & 14,3 & 24 & 70 & 76.23 \\
\hline 33 & & $\mathrm{~m}$ & 60 & Pharynx & 51,3 & 24 & 70 & 203.2 \\
\hline 34 & & $\mathrm{~m}$ & 79 & CR & 39,3 & 24 & 75 & 166 \\
\hline 35 & & $f$ & 55 & CR & 4,9 & 24 & 70 & 26 \\
\hline 36 & 2 & $\mathrm{~m}$ & 63 & Other & 4,5 & 24 & 60 & 24 \\
\hline & & $\mathrm{m}$ & 63 & Other & 62 & 22 & 70 & 120 \\
\hline 37 & & $f$ & 59 & Breast & 13,2 & 24 & 70 & 45 \\
\hline 38 & & $f$ & 66 & CR & 17,8 & 24 & 65 & 127.3 \\
\hline 39 & 2 & $\mathrm{~m}$ & 65 & BC & 54,9 & 24 & 65 & 403.6 \\
\hline & & $m$ & 65 & BC & 12,7 & 24 & 60 & 403.6 \\
\hline 40 & 2 & $f$ & 69 & Other & 27,5 & 22 & 60 & 135.6 \\
\hline
\end{tabular}




\section{Cureus}

\begin{tabular}{|c|c|c|c|c|c|c|c|c|}
\hline & & $f$ & 70 & Other & 64,7 & 22 & 70 & 416.3 \\
\hline 41 & & $f$ & 72 & GIT & 9,4 & 22 & 75 & 44.9 \\
\hline 42 & & $\mathrm{~m}$ & 37 & UGT & 5,9 & 22 & 75 & 20.7 \\
\hline 43 & & $f$ & 66 & UGT & 30,1 & 22 & 65 & 163.1 \\
\hline 44 & & $f$ & 58 & Other & 22,9 & 22 & 70 & 75.8 \\
\hline 45 & & $\mathrm{~m}$ & 68 & Other & 42,6 & 22 & 70 & 183.8 \\
\hline 46 & & $f$ & 73 & Other & 12,7 & 22 & 75 & 62.4 \\
\hline \multirow[t]{2}{*}{47} & 2 & $\mathrm{~m}$ & 59 & CR & 15,4 & 22 & 60 & 85.4 \\
\hline & & $\mathrm{m}$ & 60 & CR & 21,1 & 22 & 70 & 205 \\
\hline \multirow[t]{2}{*}{48} & 2 & $f$ & 43 & CR & 2,3 & 22 & 65 & 40.8 \\
\hline & & $\uparrow$ & 43 & CR & 7,3 & 22 & 65 & 40.8 \\
\hline 49 & & $f$ & 52 & CR & 5,0 & 22 & 60 & 32.1 \\
\hline 50 & & $\mathrm{~m}$ & 65 & Prostata & 13,8 & 22 & 75 & 85.1 \\
\hline \multirow[t]{2}{*}{51} & 2 & $\mathrm{~m}$ & 69 & other & 62 & 22 & 75 & 180 \\
\hline & & $\mathrm{m}$ & 69 & other & 49,0 & 22 & 70 & 252 \\
\hline 52 & & $\mathrm{~m}$ & 65 & Urothel & 4,2 & 22 & 55 & 22.1 \\
\hline 53 & & $\mathrm{~m}$ & 61 & other & 7,0 & 22 & 60 & 40.3 \\
\hline 54 & & $f$ & 64 & CR & 7,9 & 22 & 65 & 73.1 \\
\hline 55 & & $\mathrm{~m}$ & 70 & CR & 18,1 & 22 & 65 & 137.5 \\
\hline 56 & & $\mathrm{~m}$ & 69 & other & 1,9 & 22 & 65 & 18.4 \\
\hline 57 & & $f$ & 67 & other & 13,6 & 22 & 65 & 125.8 \\
\hline 58 & & $f$ & 62 & other & 50,5 & 22 & 60 & 200.9 \\
\hline 59 & & $\mathrm{~m}$ & 62 & other & 8,4 & 22 & 70 & 45.6 \\
\hline \multirow[t]{2}{*}{60} & 2 & $f$ & 41 & GIT & 4,1 & 22 & 60 & 24 \\
\hline & & $f$ & 41 & GIT & 4,1 & 22 & 60 & 24 \\
\hline 61 & & $f$ & 65 & Sarkoma & 33,4 & 22 & 70 & 231.5 \\
\hline 62 & & $f$ & 53 & $B C$ & 65 & 22 & 65 & 210 \\
\hline
\end{tabular}

\section{TABLE 1: Patient series}

Abbreviations: $\mathrm{f}=$ female, $\mathrm{m}=$ male, $\mathrm{No}=$ number, $\mathrm{BC}=$ bronchial carcinoma, $\mathrm{GIT}=$ gastrointestinal tract other than colorectal, $\mathrm{CR}=$ colorectal, UGT = urogenital-tract, $10 \mathrm{~Gy}=$ volume of the tissue surrounding the lesion receiving $10 \mathrm{~Gy}$ and more. 
All patients were evaluated by a thoracic surgeon, a radiation oncologist, and an interventional radiologist for eligibility of treatment and fiducial placement. Forty-two patients received one percutaneously implanted gold fiducial into the tumor using a pre-loaded 19-gauge needle under CT guidance and local anesthesia [11]. No immobilization device was used during treatment. Patients underwent contrast-enhanced CT scanning through the entire thoracic cavity using $1 \mathrm{~mm}$ thick slices. The treating surgeon and the radiation oncologist identified the location of the tumor, and a radiosurgical treatment plan was developed using a non-isocentric, inverse-planning algorithm. The last 23 patients were planned by means of Monte Carlo calculation which did replace the former ray tracing algorithm just recently. The tumor was outlined in sequential axial CT images. The PTV was defined as the CTV, plus $6 \mathrm{~mm}$, margin to account for microscopic tumor infiltration. In order to reduce set-up error, patients were first aligned to the nearest vertebra to the tumor using fiducial-free spinal registration software (Xsight $^{\mathrm{TM}}$, Accuray Incorporated) [12]. After this set-up procedure, the lung treatment plan was loaded for treatment delivery. Modelling to correlate tumor motion (fiducial marker) and respiratory motion (LED on chest wall) was performed. We aimed to achieve a correlation error of less than $1 \mathrm{~mm}$ to start treatment. Radiation was then delivered in a single fraction using 24 Gy prescribed to the 60 - 80\% isodose and $22 \mathrm{~Gy}$ in cases with Monte Carlo dose calculation. If breathing patterns changed during the course of treatment, exceeding the recommended correlation error, remodelling was performed. Patients were followed clinically and radiographically after radiosurgery in two-month intervals.

\section{Results}

\section{Respiratory motion compensation}

All patients could be treated technically using the real-time respiratory tumor tracking technique. Our approach to implant only one fiducial and to minimize the set-up error by aligning the patient with the non-invasive spinal protocol proved to be feasible, safe, and welltolerated by the patient. Remodelling was necessary according to the breathing patterns of the patients. One-to-five remodelling procedures (mean two) during the treatment cycle became necessary. The median treatment time was one hour 10 minutes (30 minutes - two hours).

\section{Clinical outcome}

Follow-up ranged from two weeks to 30 months (median: 10 months). Thirteen patients had died during the follow-up period. The six, 12-, 18- and 24-months actuarial overall survival rates after SRS were $0.92,0.79,0.67$ and 0.61 , respectively (Figure 1 ). The six, 12 , and 24month actuarial local tumor control rates were 0.98, 0.93 and 0.93, respectively (Figure 2). Five patients had documented local failure. One of the five patients with progressive disease was treated for a recurrent tumor after surgery and conventional radiation therapy. He was originally planned for radiosurgery on the basis of a PET CT scan because standard CT could not clearly visualize the tumor recurrence. The PET CT obviously did not show the full extent of the disease. The recurrent tumor was subsequently resected. Six patients required a chest tube insertion after fiducial implantation because of pneumothorax. Four patients experienced nausea after treatment. Fifteen patients showed Grade 1 and one patient Grade 2 signs of pneumonitis. No Grade 3 or Grade 4 toxicity was detected. No other adverse events were noted. 


\section{Cureus}

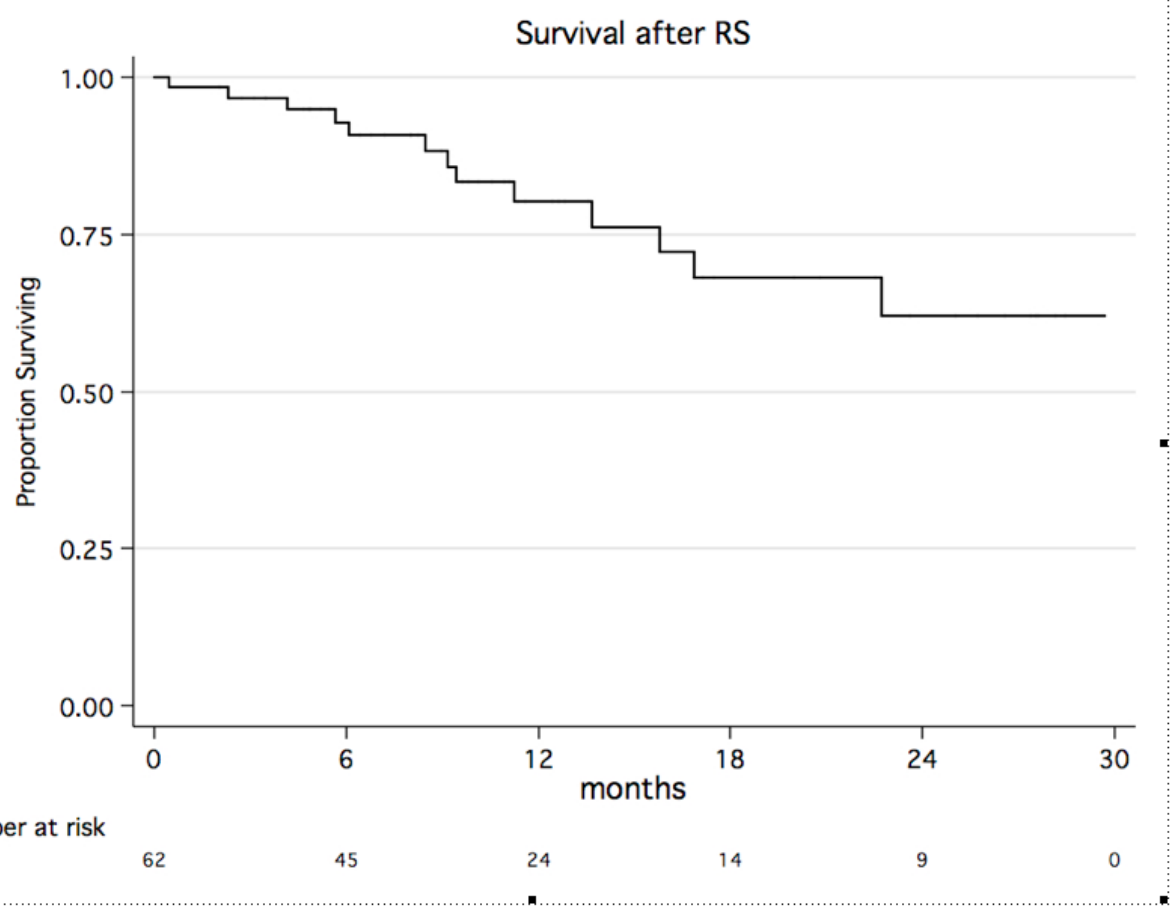

FIGURE 1: Kaplan Meier Plot showing the actuarial overall survival rate.

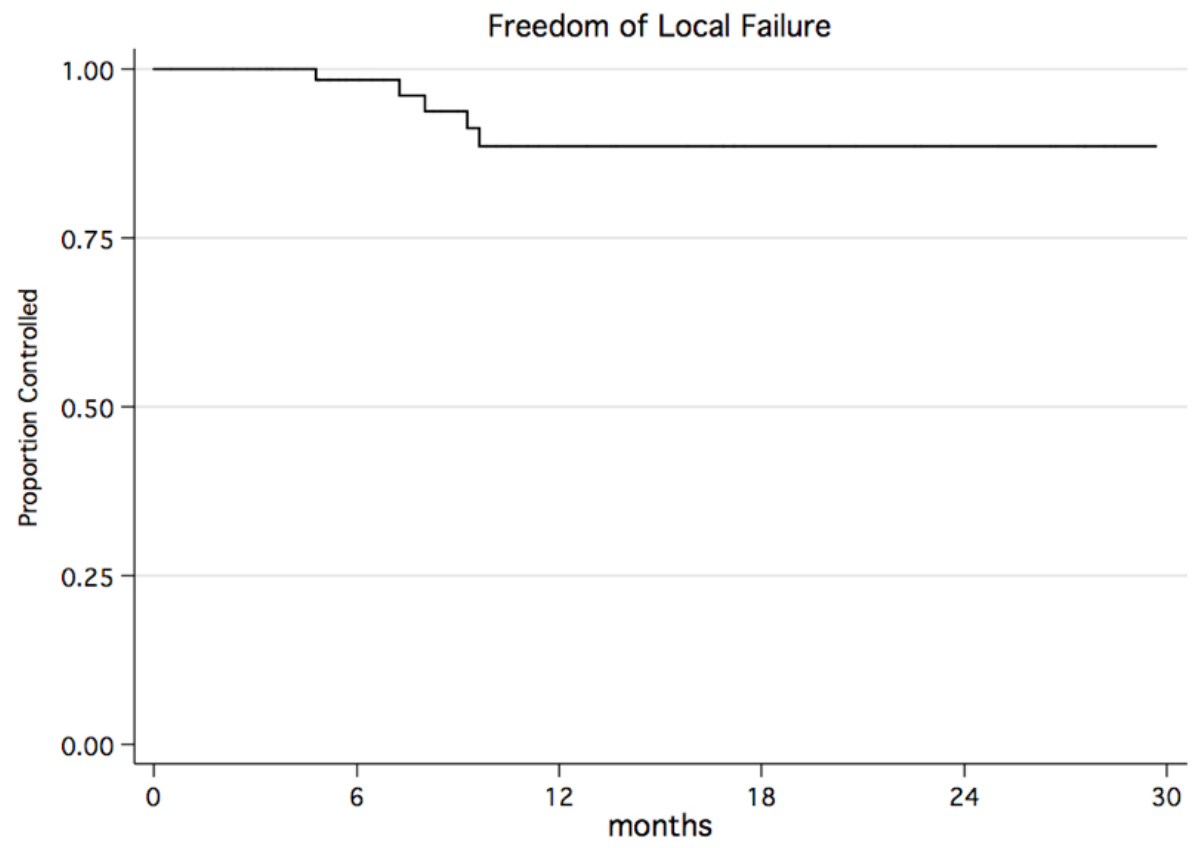

FIGURE 2: Kaplan Meier Plot showing the actuarial local tumor control rate. 


\section{Discussion}

It is well known that tumors in the lung move significantly. The movement can be craniocaudal and anterior - posterior. It has also been demonstrated that the shape of lung lesions can change with respiration [13-14]. This explains why simply applying margins around the tumor is not sufficient; the risk of missing the target and irradiating too much healthy lung tissue is high. The respiratory tracking capabilities of the current radiosurgery system overcome most of the obvious disadvantages of standard radiation therapy for lung tumors. The integration of image-guided robotics enables the patient to be treated without any fixation devices as the robot compensates for tumor movement. The system constantly tracks the lesion, detecting inaccuracies, and correcting, if indicated.

\section{Clinical application}

All selected patients could be treated with the described set-up in a single outpatient session. All patients were evaluated for feasibility of treatment by a multi-specialty team composed of thoracic surgeons, a radiation oncologist, a radiation physicist, and an interventional radiologist. It was decided to apply a homogeneous dose of $22-24 \mathrm{~Gy}$ to all patients prescribed to the 60 - 80\% isodose. This single-dose approach differs from several other published approaches to stereotactic, high-dose radiation treatment in the lung. Timmerman, et al. suggested a dose of 3 x $20 \mathrm{~Gy}$ for hypofractionated lung radiosurgery based on a Phase I dose escalation study using an extracranial stereotactic body frame [15]. He reported on one case of Grade 3 pneumonitis and one case of Grade 3 hypoxia. The concomitant prospective Phase II trial confirmed the high local control rates using this treatment regime, although about $50 \%$ patients with central lung tumors experienced severe radiation toxicity during the two year follow-up time [16]. Onishi, et al. demonstrated encouraging results with a local failure rate of only $14.5 \%$ on a multi-institutional retrospective analysis of 245 patients treated by stereotactic radiation therapy with up to 22 fractions [7]. Three published reports exist in which patients were treated with the CyberKnife for lung tumors. Only in the most recent one were all 20 patients treated with the latest version of the respiratory tracking system used in the present study [17]. The majority of the patients in the Whyte and Le publication were treated using a breathhold technique [18-19]. None of these studies determined an optimal dose prescription. Whyte, et al. delivered $15 \mathrm{~Gy}$ in a single session, and Le, et al., in a follow-up study, added two groups of patients treated with single fractions of $25 \mathrm{~Gy}$ and $30 \mathrm{~Gy}$. They found increased radiotherapy complications for doses greater than $25 \mathrm{~Gy}$ and a low one-year local control rate (54\%) for doses less than $20 \mathrm{~Gy}$. Nuyttens, et al. treated 20 patients applying 3 x $12 \mathrm{~Gy}, 3$ x 15 Gy and 3 x 20 Gy delivered every other day. Taking into account these findings and in order to overcome these highly heterogeneous dose prescription schemes, we selected a stringent treatment algorithm in which $22-24 \mathrm{~Gy}$ was delivered in single session. We hypothesized that a technology providing high accuracy for moving targets should be capable of treating a lung tumor in a manner similar to the single-session treatment often employed for brain or spine tumors. This concept is feasible as long as the tumor is clearly visible on CT and not too large. We suggest that this treatment regime be applied only to relatively small tumors (maximal diameter of $3.5 \mathrm{~cm}$ ). The dose was well-tolerated by all patients during short-term follow-up. Longer follow-up is needed for definitive clinical recommendations and risk estimations.

In order to reduce the invasiveness of the procedure to a minimum, we decided to only implant one fiducial into the lung tumor for image-guided tracking. Although one fiducial is generally regarded as insufficient for 3-D tracking, we thought the risk of pneumothorax would be unacceptably high when implanting three fiducials or more into the lung. Furthermore, as we mainly treated small tumors, it would not have been feasible to implant three or four fiducial inside these tumors -- fiducial markers placed in normal lung can easily migrate and are therefore unsuitable. To minimize the set-up error, and particularly, the rotational error, we did an automated set-up as during spine radiosurgery, aligning the patient primarily to the 
vertebrae nearest to the lesion. After this set-up, we switched to the respiratory tumor tracking algorithm for creating the correlation model between tumor motion and the respiratory motion. This procedure was well-tolerated by all patients.

Current research indicates that lung tumor tracking for peripheral tumors may be possible based on X-ray imaging alone. This is already realized with more sophisticated image processing and image registration techniques that automatically lock onto the tumor directly [10] (Xsight lung software, Accuray Inc.). This tracking innovation enables clinicians to treat patients without implanting any fiducials, potentially making lung radiosurgery a completely non-invasive procedure. In our early experience, this non-fiducial tracking capability can only be realized in tumors with a diameter over $1.5 \mathrm{~cm}$ and a peripheral location where the spine and the heart soft tissue are not overlapping with the orientation of the stereoscopic beams of the imaging system. This might be the case in about $10 \%$ of the lung cases potentially available for lung SRS. We used this technique in 10 patients and could confirm the feasibility of the tracking method.

\section{Conclusions}

Image-guided respiratory real-time tumor tracking for robotic lung radiosurgery is a technically safe and stable procedure. The single-session procedure we applied showed excellent tolerability and seems to be a feasible treatment method for selected patients with small tumors. Particularly, the fiducial-free tracking capabilities will have a significant impact on the ease of use for lung tumor radiosurgery in the near future. A longer follow-up and prospective evaluations are needed for definitive clinical results.

\section{Additional Information \\ Disclosures}

Human subjects: Consent was obtained by all participants in this study. The University of Munich Hospitals institutional review board issued approval \# 383-08. Animal subjects: All authors have confirmed that this study did not involve animal subjects or tissue. Conflicts of interest: In compliance with the ICMJE uniform disclosure form, all authors declare the following: Payment/services info: All authors have declared that no financial support was received from any organization for the submitted work. Financial relationships: Alexander Muacevic declare(s) an alternate financial activity from The European Cyberknife Center Munich. co-owner . Other relationships: All authors have declared that there are no other relationships or activities that could appear to have influenced the submitted work.

\section{References}

1. Dosoretz DE, Katin MJ, Blitzer PH, Rubenstein JH, Galmarini DH, Garton GR, Salenius SA: Medically Inoperable Lung Carcinoma: The Role of Radiation Therapy . Semin Radiat Oncol. 1996, 6:98-104.

2. Graham PH, Gebski VJ, Langlands AO: Radical Radiotherapy for Early Nonsmall Cell Lung Cancer. Int J Radiat Oncol Biol Phys . 1995, 31:261-66.

3. Jeremic B, Classen J, Bamberg M: Radiotherapy Alone in Technically Operable, Medically Inoperable, Early-Stage (I/Ii) Non-Small-Cell Lung Cancer. Int J Radiat Oncol Biol Phys . 2002, 54:119-130.

4. Kaskowitz L, Graham MV, Emami B, Halverson KJ, Rush C: Radiation Therapy Alone for Stage I Non-Small Cell Lung Cancer. Int J Radiat Oncol Biol Phys . 1993, 27:517-23.

5. Blomgren H, Lax I, Näslund I, Svanström R: Stereotactic High Dose Fraction Radiation Therapy of Extracranial Tumors Using an Accelerator. Clinical Experience of the First ThirtyOne Patients. Acta Oncol. 1995, 34:861-70.

6. Nagata Y, Takayama K, Matsuo Y, Norihisa Y, Mizowaki T, Sakamoto T, Sakamoto M, Mitsumori M, Shibuya K, Araki N, Yano S, Hiraoka M: Clinical Outcomes of a Phase I/Ii Study 
of 48 Gy of Stereotactic Body Radiotherapy in 4 Fractions for Primary Lung Cancer Using a Stereotactic Body Frame. Int J Radiat Oncol Biol Phys . 2005, 63:1427-31.

7. Onishi H, Araki T, Shirato H, Nagata Y, Hiraoka M, Gomi K, Yamashita T, Niibe Y, Karasawa K, Hayakawa K, Takai Y, Kimura T, Hirokawa Y, Takeda A, Ouchi A, Hareyama M, Kokubo M, Hara R, Itami J, Yamada K: Stereotactic Hypofractionated High-Dose Irradiation for Stage I Nonsmall Cell Lung Carcinoma: Clinical Outcomes in 245 Subjects in a Japanese Multiinstitutional Study. Cancer . 2004, 101:1623-31.

8. Schweikard A, Glosser G, Bodduluri M, Murphy MJ, Adler JR: Robotic Motion Compensation for Respiratory Movement During Radiosurgery. Comput Aided Surg . 2000, 5:263-77.

9. Schweikard A, Shiomi H, Adler JR: Respiration Tracking in Radiosurgery. Med Phys . 2004, 31:2738-41.

10. Schweikard A, Shiomi H, Adler JR: Respiration Tracking in Radiosurgery without Fiducials. Medical Robotics and Computer Assisted Surgery . 2005, 1:19-27.

11. Shirato H, Seppenwoolde Y, Kitamura K, Onimura R, Shimizu S: Intrafractional Tumor Motion: Lung and Liver. Semin Radiat Oncol. 2004, 14:10-18.

12. Muacevic A, Staehler M, Drexler C, Wowra B, Reiser M, Tonn JC: Technical Description, Phantom Accuracy, and Clinical Feasibility for Fiducial-Free Frameless Real-Time ImageGuided Spinal Radiosurgery. J Neurosurg Spine . 2006, 5:303-12.

13. Wulf J, Hädinger U, Oppitz U, Thiele W, Flentje M: Impact of Target Reproducibility on Tumor Dose in Stereotactic Radiotherapy of Targets in the Lung and Liver. Radiother Oncol. 2003, 66:141-150.

14. Wulf J, Haedinger U, Oppitz U, Thiele W, Mueller G, Flentje M: Stereotactic Radiotherapy for Primary Lung Cancer and Pulmonary Metastases: A Noninvasive Treatment Approach in Medically Inoperable Patients. Int J Radiat Oncol Biol Phys . 2004, 60:186-96.

15. Timmerman R, Papiez L, McGarry R, Likes L, DesRosiers C, Frost S, Williams M: Extracranial Stereotactic Radioablation: Results of a Phase I Study in Medically Inoperable Stage I NonSmall Cell Lung Cancer. Chest. 2003, 124:1946-55.

16. Timmerman R, McGarry R, Yiannoutsos C, Papiez L, Tudor K, DeLuca J, Ewing M, Abdulrahman R, DesRosiers C, Williams M, Fletcher J: Excessive Toxicity When Treating Central Tumors in a Phase Ii Study of Stereotactic Body Radiation Therapy for Medically Inoperable Early-Stage Lung Cancer. J Clin Oncol . 2006, 24:4833-39.

17. Nuyttens JJ, Prévost JB, Praag J, Hoogeman M, Van Klaveren RJ, Levendag PC, Pattynama PM: Lung Tumor Tracking During Stereotactic Radiotherapy Treatment with the Cyberknife: Marker Placement and Early Results. Acta Oncol . 2006, 45:961-65.

18. Le QT, Loo BW, Ho A, Cotrutz C, Koong AC, Wakelee H, Kee ST, Constantinescu D, Whyte RI, Donington J: Results of a Phase I Dose-Escalation Study Using Single-Fraction Stereotactic Radiotherapy for Lung Tumors.. J Thorac Oncol. 2006, 1:802-09.

19. Whyte RI, Crownover R, Murphy MJ, Martin DP, Rice TW, DeCamp MM Jr, Rodebaugh R, Weinhous MS, Le QT: Stereotactic Radiosurgery for Lung Tumors: Preliminary Report of a Phase I Trial. Ann Thorac Surg . 2003, 75:1097-1101. 